\title{
Coherence imaging for laser detection
}

\section{Benton, David, Sugden, Kate, Zandi, Marie}

David Benton, Kate Sugden, Marie Zandi, "Coherence imaging for laser detection," Proc. SPIE 11537, Electro-Optical and Infrared Systems: Technology and Applications XVII, 115370K (20 September 2020); doi: 10.1117/12.2573194

SPIE. Event: SPIE Security + Defence, 2020, Online Only 


\title{
Coherence imaging for laser detection
}

\author{
David Benton, Kate Sugden and Marie Zandi \\ Aston Institute of Photonic Technologies, Aston University, Aston Triangle, Birmingham, B4 7ET. UK
}

\begin{abstract}
The detection of lasers by means of coherence detection often uses interferometry in a one-dimensional manner such as with a Mach Zehnder interferometer. We are taking a two dimensional approach making use of a spatial light modulator and a camera to examine the results of binary holography in differentiating coherent and incoherent incoming light. A DMD micromirror array focusing light onto a CCD camera is presented as a method for determining the presence of coherent light, its wavelength and angle of arrival. We discuss the use of Zernike modes to overcome the aberrations inherent in the DMD device, thereby improving the focussing quality and improving the precision of wavelength determination.
\end{abstract}

\section{INTRODUCTION}

Lasers are now a pervasive technology with many familiar applications that range from communication, material processing, 3D scanning, printing, medical applications and many more. The generation of laser radiation requires such an imbalance of thermodynamic equilibrium that only highly unusual astrophysical scale phenomena can generate lasing in nature [1]. All other lasers are engineered and used with a purpose. It is the intent behind the use that drives military desire to detect lasers[2]. Military applications of lasers include range finding, target designation, laser dazzle and missile control.

In the last few years a more widespread hazard has arisen from the preponderance of continuous wave (CW) visible laser diodes - so called laser pointers. Handheld visible lasers with powers of several Watts are available for a few hundred dollars with an ever-expanding range of wavelengths. These lasers have proven to be a menace when directed towards aircraft as they approach a landing, with over 1500 reports of lasers dazzling pilots in the UK and US last year. Conventional LWRs do not perform well at detecting these lasers. $\mathrm{CW}$ lasers are therefore a specific challenge in the world of laser detection.

Wang [3] divided laser detection into three categories - coherent recognition, scattering recognition and spectrum recognition. These are categories based on what is observed rather than the discriminating characteristic. Benton [4] took a classification approach based on discrimination technique - imaging, spectral and coherence. The first two categories are both essentially discriminating based on brightness. Imaging systems make use of CCD arrays [5][6] and intensified cameras[2], whilst semi-imaging systems look for tell-tale circles from bright sources[7]. Spectroscopic systems typically use dispersion with a diffraction grating and a detector array [8][9]. Discrimination based on coherence detection is the main subject of this work.

The concept of using the coherence properties of laser radiation as its signature of detection has been around for decades with the underlying principle being that broadband background radiation has a very short coherence length, typically microns, which suppresses interference effects in interferometers which are unbalanced (asymmetric).[10-14]. Benton [14] made use of a Mach Zehnder interferometer (MZI) to produce a low-cost laser detection system capable of discriminating wavelength.

\footnotetext{
Electro-Optical and Infrared Systems: Technology and Applications XVII, edited by Duncan L. Hickman, Helge Bürsing, Proc. of SPIE Vol. 11537, 115370K

(C) 2020 SPIE · CCC code: 0277-786X/20/\$21 · doi: 10.1117/12.2573194
} 
The digital micromirror device (DMD) produced by Texas Instruments is an array of micro-electromechanical (MEMS) tilting micromirrors that have found widespread use in the consumer market of digital projection. Individual mirrors, typically 13 microns in size, pivot about a diagonal tilting between $+12^{\circ}$ and $-12^{\circ}$ thus representing on and off states. Across the mirror array binary patterns can be used to represent images. When the optical source is coherent the binary spatial pattern acts as a hologram for diffracted light. There have been many hundreds of additional and alternative applications of DMDs including beam steering[15], spectral filtering and dazzle protection[16] to name a few. This work is aiming to make use of the diffraction of coherent sources as a means of identifying and characterizing laser sources. Some valuable steps along this journey in terms of improving image quality are presented.

\section{METHOD.}

The binary nature of the DMD means that it is very well matched to the concept of the binary Fresnel zone plate (FZP) which can be used to produce a focus. This concept has been used for beam steering [17] and also as a method of wavelength determination [18]. The focal length of a FZP is controlled using the radial size of the zones. Thus a FZP written on a DMD can step through a series of focal lengths and identify a minimum spot size when viewed with a stationary camera at a known distance. Ref [18] claimed a statistical uncertainty of $0.03 \mathrm{~nm}$ when using this process. However, it is clear that the focussed spot being measured was subject to significant aberration. DMDs which reflect at $24^{\circ}$ are subject to significant astigmatism as the FZP circles look like ellipses when seen from the focus. A controllable spatial array means this can be corrected and the focus improved [17]. However the DMD system (mirror array and window) has inherent aberrations which should also be accounted for. This is suitably demonstrated by capturing an interferogram of the DMD as shown in Figure 1.

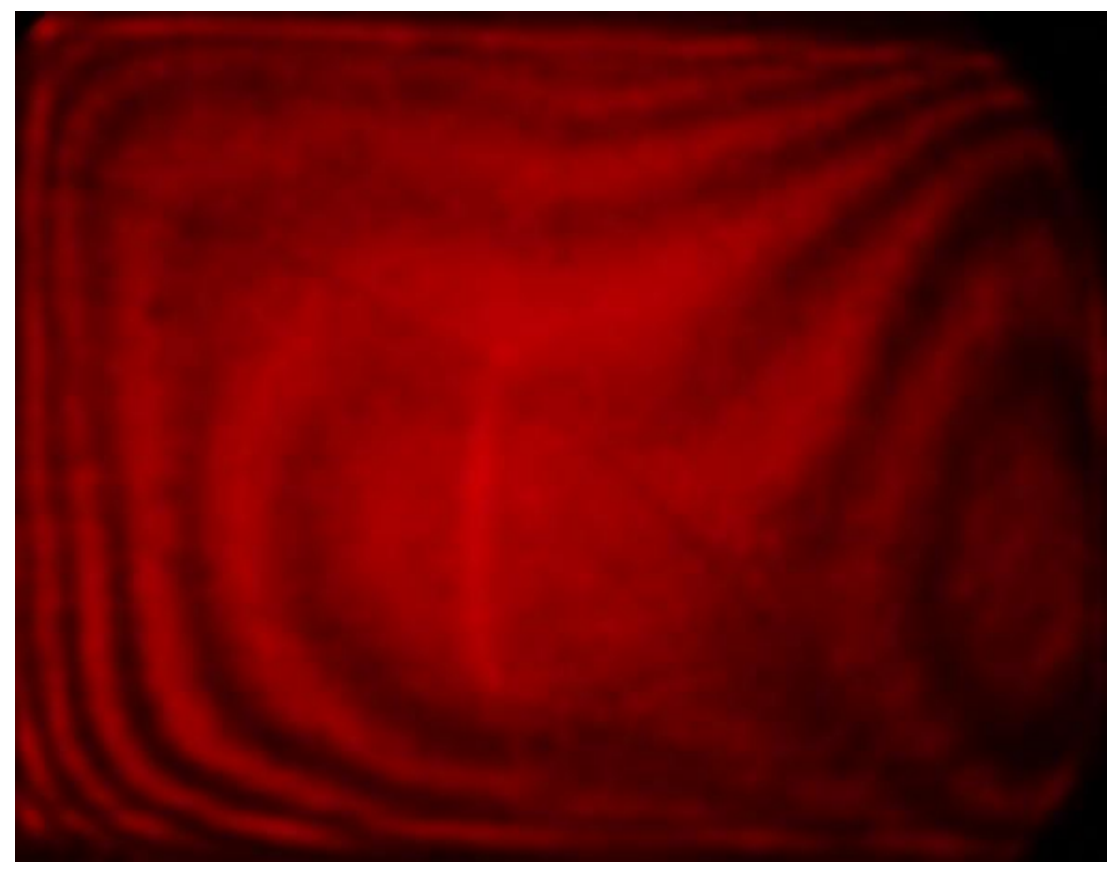

Figure 1. An interferogram of the DMD surface showing inherent aberrations. 
The effect of these aberrations can in principle be reduced by writing appropriate patterns to the DMD. Such aberrations will be present on every DMD and the work presented here can assist in overcoming these effects whatever the application. We will proceed by using the set of orthogonal Zernike modes as given in Table 1. These modes correspond to the traditional aberrations named in the final column. The parameter $r$ represents the radial distance from the centre of the DMD (normalised to the max radius) and $\theta$ is the azimuthal angle.

\section{TERM}

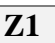

$\mathbf{Z 2}$

$\mathbf{Z 3}$

$\mathbf{Z 4}$

$\mathrm{Z5}$

Z6

Z7

$\mathbf{Z 8}$

Z9

Z10

Z11

Z12

Z13

Z14

Z15

\section{ZERNIKE NAME}

EXPRESSION

\section{Piston}

tip

tilt

Defocus

Oblique astigmatism

Vertical astigmatism

Vertical coma

Horizontal coma

Vertical trefoil

Oblique trefoil

Primary spherical

Vertical secondary astigmatism

Oblique secondary astigmatism

Oblique quadrafoil

Vertical quadrafoil

Table 1. Zernike modes

These modes are however only orthogonal for circular apertures and hence modified versions are used to account for the rectangular DMD [19]. The defocus term produces the circular rings of the FZP, the astigmatism terms produce ellipses caused by the off axis reflection and the tip and tilt terms are what can be used to steer the focussed beam as in [20].

We take an approach used with the FZP but in a more general sense, which is to only use light with one phase polarity such that pixels contribute complex amplitudes that all sum constructively. The "opaque" regions that do not contribute are directing light away from the camera. For each Zernike mode the phase at each pixel is calculated and contributed to a final phase map. Once all Zernike modes have contributed the binary amplitude of each pixel is determined based on whether the net polarity is positive or negative when calculated at the camera. Once the appropriate $\mathrm{ZM}$ amplitudes have been determined a wavelength dependent correction pattern for the DMD can be obtained by removing the defocus and astigmatism terms.

A collimated beam is incident upon the DMD and reflected towards a camera where the beam is analysed see Figure 2. An initial progression of only focus terms is applied to the DMD and the beam spot size recorded allowing the best focus position to be located for the fixed camera position 


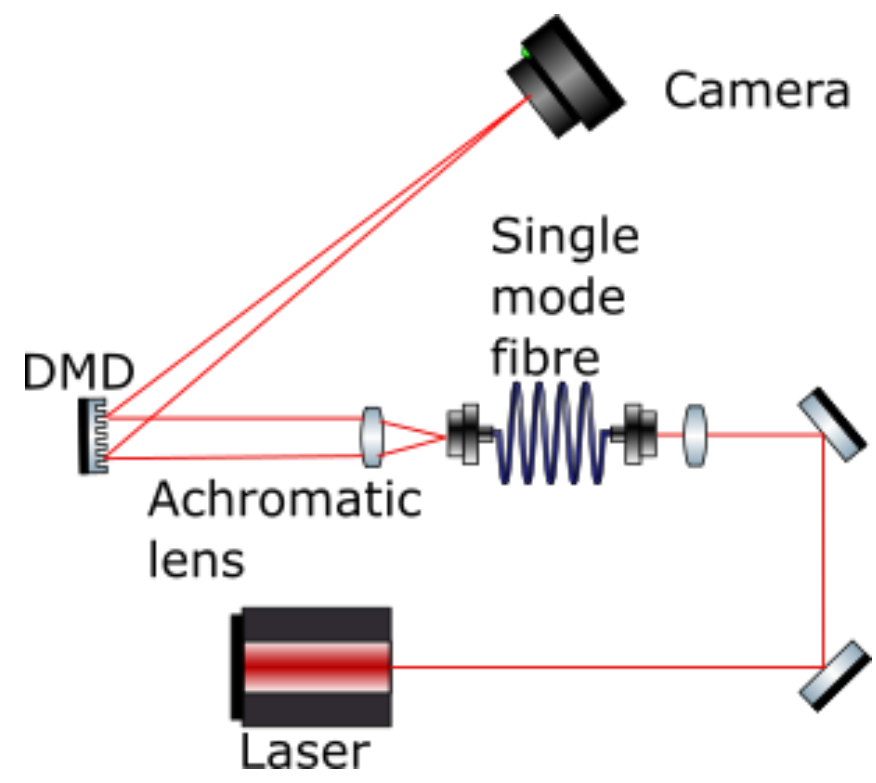

Figure 2. A schematic diagram of the system used for laser light to DMD

Two metrics are used in assessing the beam spot:

1. The spot width in both horizontal and vertical directions obtained by projecting the spot onto the $\mathrm{x}$ and $\mathrm{y}$ axes then performing a Gaussian fit.

2. The peak intensity ensuring that the camera does not saturate.

Correcting for the inherent aberrations is done by fixing the focus term such that the camera observes the circle of least confusion - so that the widths in $\mathrm{x}$ and $\mathrm{y}$ directions are equal (note intensity can be misleading due to the effects of astigmatism producing higher intensities away from the correct focal position). With focus fixed individual Zernike mode amplitudes are systematically varied to optimise the spot focus - by maximising the peak intensity and minimising the peak widths. This process completed for the first 15 Zernike modes and then iterated to compensate for any non-orthogonalities. A similar principle but different method was taken in ref [21].

With the Zernike mode amplitudes now fixed the defocus term can be systematically varied to observe the variation in spot size and locate the amplitude that provides the best focus. Using a HeNe at $633 \mathrm{~nm}$ the best focus occurred at an amplitude of 28.6 which produced a focal length of $45 \mathrm{~cm}$.

The variation in spot metrics are shown in Figure 3. It shows the change in vertical and horizontal widths of the focussed laser spot, a HeNe laser $(633 \mathrm{~nm})$, as the focus parameter is changed. For comparison data with and without Zernike mode correction has been included. Before Zernike mode correction the effects of astigmatism are large and a clear difference in best focus in the vertical and horizontal directions can be seen. The circle of least confusion is the crossing point of these plots and in the first instance is taken as the best focus position. After correction the spot size is significantly smaller. Clearly the corrections make a significant difference with the final spot size being 78 times smaller (350 pixels down to 4.5 pixels). 


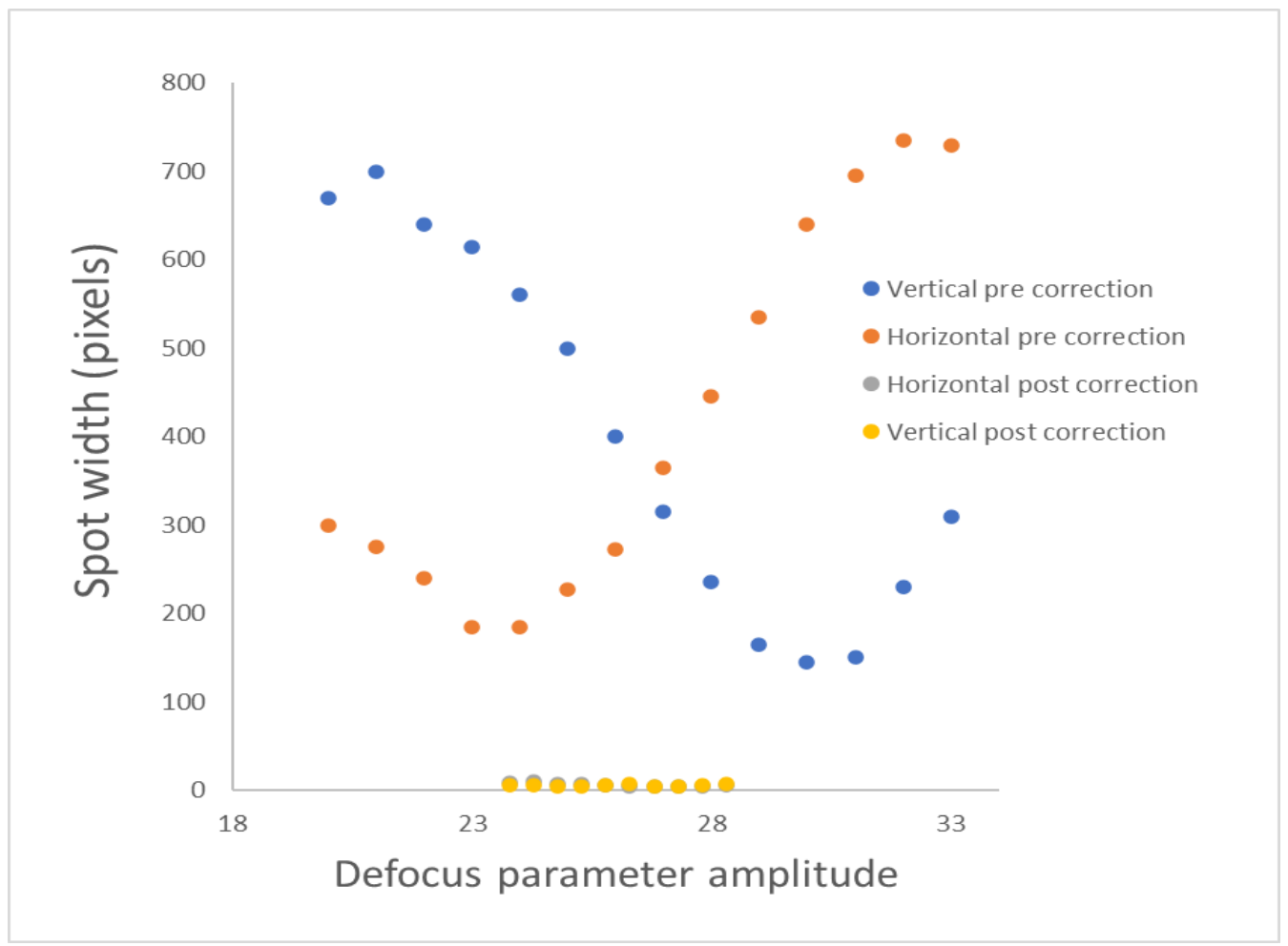

Figure 3. Focussed spot size variation as defocus parameter is varied.

The optimised amplitudes of the ZM are shown in Figure 4 along with the normalised peak intensity after optimisation. Clearly astigmatism is largest contributing aberration, but other aberration corrections are also beneficial.

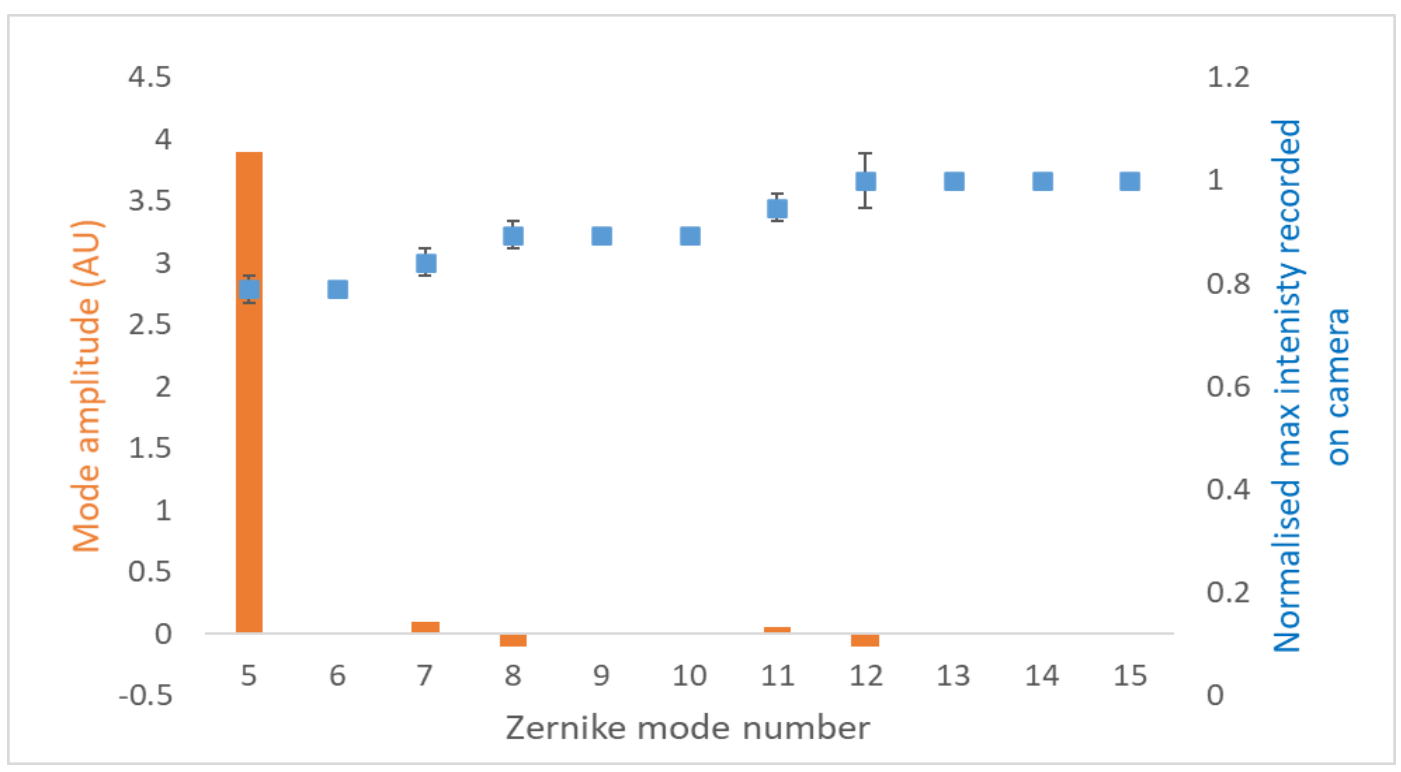

Figure 4. Zernike mode amplitudes on left hand scale and normalised spot peak intensity after applying the given mode amplitude. 
This data was completed for 3 lasers red $(633 \mathrm{~nm})$, green $(532 \mathrm{~nm})$ and blue $(405 \mathrm{~nm})$. After correction the focus variation results in much smaller, more circular focus spots and we can represent the RMS spot radius by calculating $\sqrt{\Delta x * \Delta y}$ where $\Delta x$ and $\Delta y$ are the peak widths in the $\mathrm{x}$ and $\mathrm{y}$ directions. The RMS radius values for the 3 wavelengths are plotted against focus parameter in Figure 5. In this case the actual spot width in units of $\mu \mathrm{m}$ is presented. From this data we can clearly see 2 things. Firstly the Zernike mode corrections, particularly for red and green wavelengths, display evidence of some residual aberrations. The aberration terms beyond astigmatism were corrected by optimising the detected peak intensity and this may be susceptible to problems if the astigmatism is not entirely corrected prior to optimisation. The red beam diameter is $78.5 \mu \mathrm{m}$ where the diffraction width is $35.5 \mu \mathrm{m}$ thus the beam a little over 2 times the diffraction limit. This is very likely to be improved by honing the optimisation process. Secondly there are clear differences between the wavelengths regarding the optimal defocus parameter. This should allow for rapid and reliable wavelength determination when implemented as part of a laser detection system.

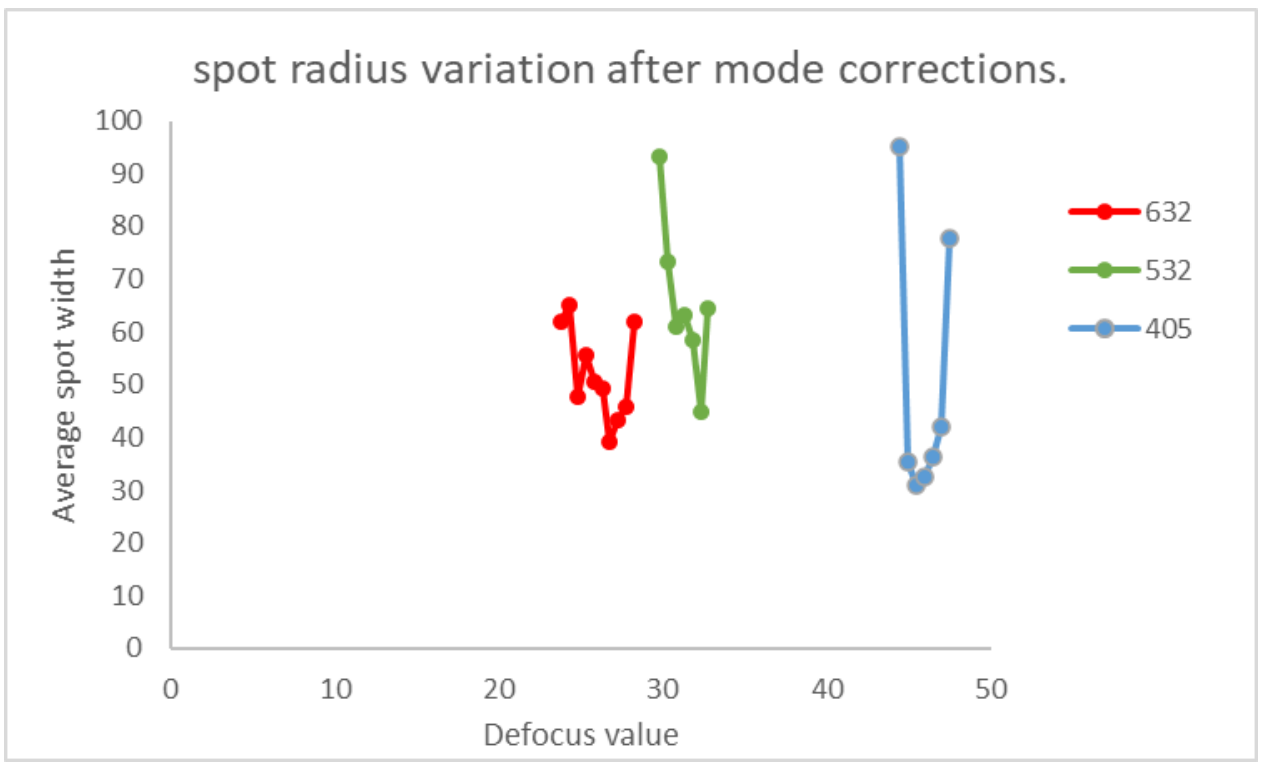

Figure 5. Spot radius variation vs defocus parameter for 3 wavelengths

\section{DISCUSSION.}

This system has been constructed so as to be as equivalent as possible for all wavelengths with the same delivery of light to the DMD. There will however be some differences, in particular with the collimation of the light striking the DMD as this will contain small differences in focussing power at different wavelengths due to inherent chromatic aberration in the achromatic lens. This is to be considered part of the system and requires calibration. There are however a number of ways to measure the wavelength with this system. Firstly the process undertaken of scanning the defocus parameter and locating the crossing point of the vertical and horizontal spot width is a feasible way of assessing wavelength but is essentially the same as ref [18]. With much improved definition it seems clear that correcting the inherent aberrations and reducing spot size will improve the sensitivity to changes in wavelength and thereby improve the potential resolution. But there is another piece of information available. The DMD is operating as a 2D diffraction grating to produce a 2D diffraction pattern. Whilst we are only considering one diffraction order, the position of that order on the 
camera is dependent upon the incident wavelength. With suitable calibration this position can be used to determine the wavelength. Such an approach requires good quality focussed spots to obtain the best resolution. An unknown laser entering the system would not be optimally focussed in such a case but the centroid of the focussed spot should give an indication of the wavelength allowing a rapid choice of focus parameter and speeding up the scanning process considerably. Once complete the best focus estimate from scanning the defocus parameter can be compared with that of the diffraction position. This work is still in progress and more work is needed to improve and determine the resolution that can be obtained with this focussing system. The principle of using a DMD opens up many possibilities for enhancing the capabilities of laser detection systems. The ability to write different patterns can enable the system to perform different tasks, such as measures to determine coherence length. Investigations of such possibilities are ongoing. The ability to significantly improve the image quality obtained by overcoming the DMDs inherent aberrations will be of benefit to many applications seeking to use the DMD in a diffractive capacity.

\section{Acknowledgements}

The authors would like to acknowledge the support of DSTL in this work.

\section{REFERENCES}

[1] Chen S. "Alien Light" https://spie.org/news/photonics-focus/janfeb-2020/astrophysical-lasers?SSO=1

[2] Dubois, J., \& Reid, F. "Detecting laser sources on the battlefield". Proc. SPIE, 6796(2007), 67962F (2007). http://doi.org/10.1117/12.779234

[3] Wang, L. et al. "Optimum design of wide angle laser detecting system based on fisheye lens and sinusoidal amplitude grating". Optics Communications, 310, 173-178 (2014).

[4] Benton, D. M., Zandi, M. A., \& Sugden, K. (2019, October). "Laser detection utilizing coherence". In Technologies for Optical Countermeasures XVI (Vol. 11161, p. 111610G). International Society for Optics and Photonics

[5] Kumar, S. et al. "Design of a laser-warning system using an array of discrete photodiodes - part I". Journal of battlefield technology 14.1 (2011).

[6] Ying, J. and Zhou, Z. "Study on Image Processing Technology in Imaging Laser Detection System”. IEEE (2010).

[7] Tipper, S., Burgess, C., \& Westgate, C. (2019, May). "Novel low-cost camera-based continuous wave laser detection". In Situation Awareness in Degraded Environments 2019 (Vol. 11019, p. 110190B). International Society for Optics and Photonics

[8] McAulay, A. D. "Detecting modulated lasers in the battlefield and determining their direction". Proc. SPIE, 7336, 73361J. (2009). https://doi.org/10.1117/12.819423

[9] Zhang, J., Tian, E., \& Wang, Z. "Research on laser warning receiver based on sinusoidal transmission grating and high speed DSPs". WSEAS Transactions on Circuits and Systems, 5(8), 1366-1371. (2006). https://doi.org/10.1088/1742-6596/48/1/152

[10] Crane Jr, R. "Laser detection by coherence discrimination". Optical Engineering, 18(2), 182212. (1979).

[11]Hickman,D. “An optical sensor based on temporal coherence properties,” J. Sci. Instrum. 21, 187-192 (1988). 
[12] Coutinho, R. C., French, H. A., Selviah, D. R., Wickramasinghe, D., \& Griffiths, H. D. "Detection of coherent light in an incoherent background [for IRST]". In 1999 IEEE LEOS Annual Meeting Conference Proceedings. LEOS'99. 12th Annual Meeting. IEEE Lasers and Electro-Optics Society 1999 Annual Meeting (Cat. No. 99CH37009) (Vol. 1, pp. 247-248). IEEE. (1999, November)

[13] Duffey, C. J. and Hickman, D. "An imaging system based on temporal coherence differences," J. Phys. D: Appl. Phys. 21, S56-S58 (1988).

[14] Benton, D.M. "Low-cost detection of lasers". Opt. Eng. 56(11), 114104 (2017).

[15]Lindle, J. R., \& Watnik, A. T. (2018). Large angle nonmechanical laser beam steering at $4.6 \mu \mathrm{m}$ using a digital micromirror device. Optical Engineering, 57(02), 1. https://doi.org/10.1117/1.OE.57.2.027108

[16]Ritt, G., \& Eberle, B. (2015). Automatic Laser Glare Suppression in Electro-Optical Sensors. Sensors, 15(1), 792-802. https://doi.org/10.3390/s150100792

[17]D. M. Benton. "Multiple beam steering using dynamic zone plates on a micromirror array". Opt. Eng.57 (2018)

[18] Mohagheghian, M., \& Sabouri, S. G. (2018). Laser wavelength measurement based on a digital micromirror device. IEEE Photonics Technology Letters, 30(13), 1186-1189. https://doi.org/10.1109/LPT.2018.2838262

[19]Dai, G. ming, \& Mahajan, V. N. (2006). Orthonormal polynomials in wavefront analysis: Error analysis. Optics InfoBase Conference Papers, 24(9), 2994-3016. https://doi.org/10.1364/fio.2006.jsua9

[20] Benton, D. M. (2017). Multiple beam steering using dynamic zone plates on a micromirror array. Optical Engineering, 57(7), 1-13. https://doi.org/10.1117/1.OE.57.7.073109

[21] Stirling Scholes, Ravin Kara, Jonathan Pinnell, Valeria Rodríguez-Fajardo, and A. F. (2020). Structured light with Digital micromirror devices: guide to best practice. Optical Engineering, 59(4), 041202. https://doi.org/10.1117/1 